\title{
A Quantum State Scenario for Biological Self-Replication
}

\section{Robert Englman}

Ariel University, Ariel, Israel

Email: englmanrobert@gmail.com

How to cite this paper: Englman, R. (2021) A Quantum State Scenario for Biological Self-Replication. Open Journal of Biophysics, $11,159-176$

https://doi.org/10.4236/ojbiphy.2021.112005

Received: January 19, 2021

Accepted: March 8, 2021

Published: March 11, 2021

Copyright (c) 2021 by author(s) and Scientific Research Publishing Inc. This work is licensed under the Creative Commons Attribution International License (CC BY 4.0).

http://creativecommons.org/licenses/by/4.0/

\begin{abstract}
With the prevalent conception of self-replication (SR, a hallmark of living systems) as a non-equilibrium process subject to thermodynamic laws, a complementary approach derives the low energy quantum states arising from a Hamiltonian that appears to be specific for bio-systems by its containing some strongly binding terms. The bindings attract properties of the template ( $\mathrm{T}$ ) and the reactants to form a replicate (R). The criterion for SR that emerges from the theory is that second order (bi-linear) interaction terms between degrees of motion of $\mathrm{T}-\mathrm{R}$ and the thermal bath dominate negatively over a linear self-energy term, and thereby provide a binding between the attributes of $\mathrm{T}$ and $\mathrm{R}$. The formalism (reminiscent of the Kramers-Anderson mechanism for superexchange) is from first principles, but hinges on a drastic simplification by modelling the $\mathrm{T}, \mathrm{R}$ and bath variables on interacting qubits and by congesting the attraction into a single (control) parameter. The development relies on further simplifying features, such as Random Phase Approximations and an Effective Hamiltonian formalism. The entropic balance to replication is considered and found to reside in the far surroundings.
\end{abstract}

\section{Keywords}

Self-Organization, Living Systems, Protein Conformations, Bifurcation, Curie-Weiss Spin Model

\section{Introduction}

It is remarkable that the concept and process of self-organization feature in so many different major disciplines [1]: in Applied Mathematics (Robotics, pioneered by von Neumann [2] and also [3]), in Physics (by a thermodynamic viewpoint, e.g. [4]) in Chemistry (primarily Organic Chemistry, and specifically Dynamic Combinatorial Chemistry [5] [6] and also in Crystallography) and, 
notably, in Biology (e.g., DNA copying as in meiosis, mitosis or binary fission, in cell divisions [7] [8] [9]). With a net so widely spread, it cannot be expected to be doing justice to all the work done heretofore.

While addressing the subject with methods in physics, the inquiry takes us to Biology, and particularly to a specific phenomenon of self-organization, namely, self-replication (SR) [6], existing in a large variety of biological entities and recognized as one of the main marks distinguishing animate from non-animate matter. (Though, there may be rare instances of SR also in the inorganic world, but not as a cyclic process [10], or in auto-catalysis but lacking the specificity of SR [11].) Notably in Biology, SR occurs at enormously differing size scales, ranging from large animals to tiny biomolecules. Accounts of real life and laboratory occurrences of SR can be found in [11] [12], including experiments at synthesising self-replication by Leslie Orgel, Rebek, von Kiedrowski [13] [14] [15] and others, as well as template-guided nonenzymatic SR, the latter also characterizing primordial RNA replication [16]. Past theoretical works, including Stuart Kauffman's set autocatalysis, have been summarized in [17] [18], with a mathematical framework that addressed in particular the citric acid - >CO and the formose (formaldehyde - > sugar) reactions.

We have made it our aim to connect up biological SR with Physics and that at a most basic level. The price that we pay for the temerity of such undertaking is doing the job in an extreme schematic and simplified fashion, thereby producing only a preliminary investigation, to be hopefully followed by further more specific and detailed works.

Remarkably enough, the guiding idea of the present approach (though arrived at independently by us) had already been given expression in a work remote from basic Physics, in a review of Dynamic Combinatorial Chemistry (DCC) dealing with the autonomous formation of receptors, that read: "Addition of a guest (a template molecule in the terminology of the present study, RE), which binds strongly (my italics) to one of the libraries (available components, RE) shifts the equilibrium towards its formation (of a new creature or offspring, RE), resulting in the amplification (renewed formation, RE) of the successful receptor at the expense of the less successful" [5]. The italicised words "binds strongly" are the leitmotif of the present work, as will be apparent in the sequel. Even more supportive is the concluding sentence of the same reference: "We are confident therefore that DCC will engender generations of ingenious concepts and lead to new, as yet unpredictable theories". Yet closer to the approach here taken is the assertion made about 70 years earlier than that: "The living organism seems to be a macroscopic system which in part of its behaviout approaches to that purely mechanical (as contrasted with thermodyamic) conduct to which all systems tend, as the temperature approaches absolute zero ...” ([19], Chapter 6). It seems that we are at liberty, and not in conflict with the author, to qualify "mechanical" by quantum mechanical, as will be argued next. Regarding the quantum mode of the formalism, this is analogous to Kramers' and P.W. An- 
derson's mechanism for superexchange [20] [21], with the bath replacing the bridging anion and exploitation of the randomness of the former. [Note the "attractive coupling" expression (based there on third order perturbation) of Anderson following his Equation (19) in [21].]

\section{A Quest for a Quantum Description}

At the foundation of all forms (situations, states, phenomenologies) and processes (changes, developments, steady existence) that constitute our daily life lies Quantum Mechanics (QM), mostly expressed in some or other form of the Schrödinger equation, erstwhile involving a Hamiltonian and some boundary initial conditions. In most complex issues where the QM path is not feasible, Thermodynamics (Td) or Statistical Mechanics comes to the rescue and one must hope that they (QM and $\mathrm{Td}$ ) are compatible, as seems to be the case. The merging of the two, essentially in the direction of $\mathrm{QM}->\mathrm{Td}$, has become a major recent objective of research, going under the name of Eigenstate Thermalization Hypothesis [22] [23].

Life (and more precisely, biological processes), though an extraordinary and unique phenomenon, ought not to be an exception to a derivation of its thermodynamics from quantum theory, as has been the case in e.g., light production or lasing. By my understanding, a quantitatively specific description of life starting at the QM level does not exist, not even within the broad insights provided by Schrödinger [19], Bernal [24], Prigogine [25], Dyson [26] and Ruelle [27], among others.

In the following, the subject will be self-replication (SR) in biological systems, a process which is both ubiquitous and perennial. The systems in view range in their shape and size from animals (humans included) through cells to biological micro-molecules; from a Physics viewpoint every single entity in a particular system is characterized by its momentary state, inclusive of its eigen-state, orientation and location. In the following schematic and maximally simplified treatment all these varied systems are represented in one single model and all their properties are subsumed under a single yes-no alternative. (A classical, non-quantal description might lead to qualitatively similar results, but without the sharpness consequent upon quantization, e.g., of spin alignment.) Of late, SR was treated by J. England [28] [29] from a thermodynamic viewpoint. Though Ruelle [30] has formalized that work in a quantum setting, the subject still awaits a microscopic model, like the one proposed here.

The conceptual innovation of this work lies in that, contrasting to the currently prevalent understanding of replicative and other processes in presently existing living systems, as due to the background presence of enzymes that lower and raise barriers to initiate and perpetuate modifications (Figure 3 in [28] and P. Gaspard's remark in [30]), but whose dynamical variables are absent from the formalism, these are here included in an essential, though only schematic way. It should be emphasized that this work aims at providing a descriptive account of 
how SR emerges in a quantum mechanical setting, rather than why the settings occurs in systems that exhibit SR. Specifics are in section 4.1.

The article starts in section 2 with a list of the dramatic personae which enumerates the entities that take part in a real self replication event, a list that is followed by another, section 2.1, in which each biological component is schematically represented by a qubit (half-spin). The enumeration of components matches, but is more specific than those described by Ruelle [30]. (e.g., his " $X$ " are here sub-listed as $T, R, L /(T, R)$.) In subsection 3 the present approach is placed in a perspective of standard scenarios of enzymatically activated biologocical activities, pinpointing how the former is complementary to the latter.

The model Hamiltonian is given in section 4, followed by a curt but important derivation of the "control" term in subsection 4.1. The entropic state of the system is given in section 5, both before (subsection 5.1) and at the conclusion (subsection 5.2), of the replication process. The numerical results are displayed in section 6 with the simple Figure 4 .

As a side line, this work points tentatively, and on the basis of the results, at a partial answer to the quintessential question "what discriminates between animate and non-animate matter?" as contained in section 6.1. (It should be stressed that the criteria derived from this work are not in opposition to general characterisations of life, [19]-[26], but give them here an expression within a basic, Hamiltonian formalism.) Subsection 6.2 elucidates the energy scales used, followed by a Conclusion section 7. The Supplementary Information (SI) informs how the tracing in a highly entangled system is to be performed. The theorems there may have a wide application.

\section{The Biological Components}

1) The template (T), which duplicates and maintains its state during SR.

2) The library $(L)$ of available molecules, which is composed of

a) the template's counterparts, the reactants (designated $r_{1}, r_{2}, \cdots$ ), i.e., those molecules that are gathered up by $\mathrm{T}$ from just anywhere in the library ultimately to be the replicate " $R$ ", and

b) those that are unselected, but may play some role (e.g., as enzymes) in SR. These do not replicate (by the "Central Dogma" of biology).

3) The broader environment, which adjusts in the course of replication and constitutes a thermal bath supplying the needed free energy [7].

\subsection{Representation}

In our formalism, these complex structures are condensed into single qubits (1/2-spins) and their manifold intrinsic characterizations take the simplified form of just two alternatives, "up" and "down". To reproduce the roles that 1) 3) play in reality, as above, we construct a Hamiltonian in which there are (as "degrees of freedom"): 
1) A "template" qubit, whose state-stabilization is ensured by having a self-Hamiltonian of a Zeeman term form $-e_{T} \sigma_{z}^{T}$ with a very large energy pre-factor $\left(e_{T} \gg 1\right.$, in our energy units, $\sigma$ 's here and in the sequel being Pauli spin-matrices).

2a) A single potential replicate $(R)$ qubit with Zeeman energy $\approx 1$, but also having an additional interaction term with the template-qubit of the form $-W \sigma_{z}^{T} \sigma_{z}^{R}$, in which $W \gg 1$. $W$ serves as the control parameter, or "control" (for brief), in that it regulates the transition across the SR divide. The derivation of $W$ by second order perturbation is the focal point in the theoretical development.

2b) Additional qubits ( $N-2$ in number), which, together with the former two, interact through Curie-Weiss type $x$ couplings $\sigma_{x}^{i} \cdot \sigma_{x}^{j},(i, j=1, \cdots, N)$, with varying strengths, all of the order of unity. Their weights in the low-lying wave functions are minimally affected by replication.

3) A thermal bath, in weak interaction with the former, bringing about thermalization and perturbationally enhancing the replication (alignment).

\section{Scenarios for SR}

This section examines the relation of the present quantum state formalism in an SR process to other scenarios in which SR arises as an enzyme coordinated barrier jumping event. Several theories for barrier jumping in enzymatic actions are described in [31], which also amplifies the common formulation of the process along a single reaction coordinate mode by the addition of a "promoting" vibrational mode to form a two dimensional landscape of the potential energy surface. Interestingly, a "promoting mode" of opposite symmetry to accepting modes entered previously the theory of vibronic (vibrational-electronic) decay in large molecules [32].

The following figure (Figure 1) serves to visualise the complementary roles played by the two schemata for SR as transitions between two parabolic Potential Energy Surfaces (PES), whose profiles are drawn along a single reaction coordinate. The simple parabolic depiction of the biological entities does not do justice to the complexity and multiplicity of sub-conformational wells in real systems, but serves as a base. In Figure 1(a), PES are drawn on the left (in black) for the pre-SR configuration, template $T$ and pre-replicated reactants $r_{1}+r_{2}+\cdots$, and on the right (in red) for the post-SR configuration, template $\mathrm{T}$ and the replicate $\mathrm{R}$ in which the reactants have merged, with a separation barrier in-between. To initiate a left-to-right transition, the barrier is transiently lowered, shown by the short curly line and a promoting coupling between the configurations (not shown) is brought into play. This summarizes the barrier jumping scenario of $\mathrm{SR}$, in which the quantum states involved both in the ascent to the transition state and in the descent from it are not specified.

In the adjacent Figure 1(b), illustrative of the present, quantum state scheme, PES are plotted, for the situations in which the Hamiltonian is without a control term ( $W=0$, upper drawing) and one with a large control ( $\gg 1$, lower drawing). 


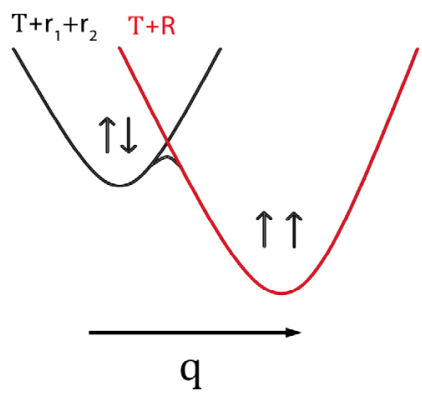

(a)
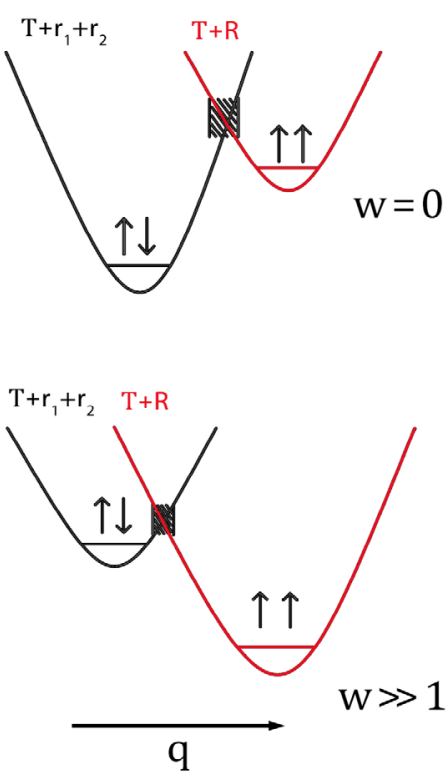

(b)

Figure 1. Alternative scenarios for SR. (a) A barrier jumping mechanism. Potential energy surfaces (PES, in arbitrary units) before self-replication for the molecular components in a $\mathrm{T}+\mathrm{r}_{1}+\mathrm{r}_{2}+\cdots$ configuration (black) and for the replicated configuration $\mathrm{T}+\mathrm{R}$ (red) against a reaction coordinate (in arbitrary units). The curved line represents a temporarily lowered barrier, across which the transition takes place. (b) Quantum level formalism. The lowest levels are schematically indicated by short horizontal lines at the nadirs of the lower PES parabolae plotted against a reaction coordinate. The PES drawn by dashed and full lines are for different Hamiltonian $(W=0$ and $W \gg 1)$ and in each case they are shown schematically for the two configurations $\left(T+r_{1}+r_{2}+\cdots\right.$ in black and $T+R$ in red). The identification of alignment and anti-alignment in the spin model with the respective red and black PES are shown by vertical arrows. Events in the shaded region are not part of this formalism.

For each Hamiltonian alternative configurations $\left(\mathrm{T}+\mathrm{r}_{1}+\mathrm{r}_{2}+\cdots\right)$ and $(\mathrm{T}+\mathrm{R})$ exists (and are drawn in their respective colouring, as before), but their low energy states (indicated by the short horizontal curves at the bottoms of the parabolae) change radically. (The two alternative configurations are formalized in our two spin model by $\uparrow \downarrow$ and $\uparrow \uparrow$, respectively, also shown in the drawings.) The source of the energy change is the increase of the control term coefficient $\mathrm{W}$ in the Hamiltonian from 0 to a large value. (The physical cause of this change is not clear; it is presumed to have an enzymatic source.) Whereas at low temperatures the resultant configuration is both determined and described by the ground state, the interim quantum states are not part in the theory. Their exclusion from the theory is indicated by the shaded regions in the figure at barrier heights: the events in this region taking place during SR are better viewed within the barrier jumping scenario.

The control W, which is thus the pivotal source of the present complementary scheme, is derived by a straightforward quantum mechanical procedure in subsection 4.1. 


\section{Formalism}

The foregoing processes are entirely rooted in the following Hamiltonian (written in arbitrary energy units)

$$
H=H(N)+H_{b a t h}+\delta H_{N-b a t h}
$$

in which the first term for $N$ interacting spins (described immediately below) is fully treated, the second term, the bath Hamiltonian is not required for our considerations, while the third, interaction term is dealt with perturbationally in the following subsection 4.1. Within this model of $\mathrm{N}$ fully interacting $1 / 2$-spins (qubits), the spins labelled $1, \cdots, N-2$ are identified with the background items $2 b^{\prime}$ in the above enumeration, (these do not replicate and are not directly subject to SR while being part of the "library"); next, a spin labelled $N-1$ which is the template (“T”, 1 ') in the above and, finally, labelled $N$ (and designated " $R$ " for "replicate", $2 \mathrm{a}$ ') is the spin that is being converted in SR to become the facsimile of the template. (The peculiar ordering of the labels aims at a simplified formulation of the "reservoir tracing", which is the concern of the Supplementary Information.) The following Hamiltonian applies to this spin system:

$$
H(N)=-\sum_{i=1, \cdots, N} h_{i} \sigma_{z}^{i}-\sum_{i, j=1, \cdots, N} g_{i, j} \sigma_{x}^{i} \sigma_{x}^{j}
$$

The Zeeman energy parameters differ in their magnitudes, as follows:

$$
i=1, \cdots, N-2: h_{i}=O(1) ; i=N-1: h_{T} \gg 1 ; i=N: h_{R}=O(1)
$$

For the inter-spin coupling we have chosen

$$
g_{i j}=O(1)(i, j=1, \cdots, N)
$$

Further details on the chosen value of the coupling coefficients are found in [33]. These are not here reproduced, as these details are not essential to the following for which the important features in the Hamiltonian are the template binding term $-h_{T} \sigma_{z}^{T}$ and a correction term hereby derived.

\section{Control}

For $\delta H_{N-b a t h}$ we postulate a bilinear form:

$$
\delta H=\sum_{b}\left(k_{b T} \sigma^{b} * \sigma^{T}+k_{b R} \sigma^{b} * \sigma^{R}\right)
$$

with $\mathrm{T}$ and $\mathrm{R}$ labelling the template and replicate spin-entities and $b$ indexing the bath qubits. ${ }^{\star}$ denotes vectorial inner product. Calculating its perturbational effect on the energy of the combined T-R-bath wave-function $\Psi$, one may assume that due to the random nature of the bath the first order diagonal perturbational correction $\langle\Psi|\delta H| \Psi\rangle$ vanishes. Regarding the second order energy correction, we first of all note that for low lying states the energy correction is predominantly negative. Next we perform a number of manipulations (approximations) that will end up by supplying an addition to the Hamiltonian $H(N)$ above of the form $-W^{\prime} \sigma_{z}^{T} \sigma_{z}^{R}$, with $W^{\prime}$ large and negative. 


$$
\begin{aligned}
\delta^{(2)}= & \Sigma_{e x c} \frac{\left|\left\langle\Psi|\delta H| \Psi_{e x c}\right\rangle\right|^{2}}{E-E_{\text {exc }}} \\
\approx & -|\delta E|^{-1} \Sigma_{e x c}\left(\left\langle\Psi|\delta H| \Psi_{e x c}\right\rangle\right)^{2} \\
\approx & -|\delta E|^{-1}\left\langle\Psi\left|(\delta H)^{2}\right| \Psi\right\rangle \\
= & -|\delta E|^{-1} \Sigma_{b}\left\langle\langle\Psi|\left[k_{b T} \sigma^{b} * \sigma^{T}\right]^{2}+\left[k_{b R} \sigma^{b} * \sigma^{R}\right]^{2}\right. \\
& \left.+2 k_{b T} k_{b R} \sigma^{b} * \sigma^{T} * \sigma^{b} * \sigma^{R}|\Psi\rangle\right\rangle
\end{aligned}
$$

In passing from the first to the second line all energy denominators involving excitational energies $E_{e x c}$ were approximatively replaced by a constant and negative mean value, thus enabling by the closure property to reach the next stage, in which appears the expectation value of $(\delta H)^{2}$. Opening this, we are led to a summation over bath entities $b$. At this stage, cross-terms in the bath indices were deemed to vanish by the randomness of phases in the bath spins. Subsequently, after opening up $(\delta H)^{2}$ in line four, the two squared terms contribute only constants to the energy and may be ignored. We are thus left with the cross term coupling target and replicate spins bi-linearly. Assuming identical signs for the coupling coefficients $k_{b T}$ and $k_{b R}$, each term in the last expectation sum contributes negatively. Adopting the so named "Effective Hamiltonian" procedure in electron spin resonance (ESR) theory, the operator between the bra-kets can be appended to the Hamiltonian $H(N)$ in Equation (2) as $-W^{\prime} \sigma_{z}^{T} \cdot \sigma_{z}^{R}$ with $W^{\prime}$ given by

$$
W^{\prime}=\frac{2}{|\delta E|} \Sigma_{b} k_{b T} k_{b R}>0
$$

The magnitude of $W^{\prime}$ is a matter of further investigation and modelling of the bath, but it may be noted that it is a sum of many terms, in principle numbering the molecular contents of the bath. There may be analogous terms, involving $x$ and $y$-spin components which are non-diagonal and presumably play a role in the energy exchange between the bath and the T-R system. They have no role in the model.

Summarizing the foregoings, we have identified within the T-R-bath-system Hamiltonian a potential source for the strongly negative bilinear coupling of the form

$$
-W \sigma_{z}^{T} \cdot \sigma_{z}^{R}
$$

in which $W=W^{\prime}+g_{T R}$ is the control parameter for self-replication.

The above approximative procedure of eliminating the bath variables due to their random (and uncorrelated) phases appears to be justified at the present level of treatment, in which the bath degrees of freedom are not dynamic i.e. not subject to equations of motion; alternative justifications rooted in the sheer size of the number of bath coordinates were also given). [In a more elaborate treatment inclusive of bath dynamics the foregoing manipulation of $(\delta H)^{2}$ 
would have been done in reverse: by tracing over the $N$-variables (the total system minus the bath) and obtaining an "Effective Hamiltonian" representing interaction within the bath coordinates, inducing correlations and entropy increase, for which predictions have already been made in [34] [35]. By their estimate, this occurs mainly well after the SR process, thus allowing one to treat the process of SR itself at the level here adopted.]

\section{Stages of Replication}

In this conceptual stages are meant, as in a "gedanken experiment", not chronological ones. For the latter, the states of bifurcation, appearing in Figure 4 as a diagonal line, may well be relevant, representing states of meta-stability.

\subsection{Pre-SR}

The control is off, $W=0$, and the thermalisation with surrounding is as yet absent. So we may take the template spin $N$ to be "up", and the library spins, excepting the potentially replicate spin $N-1$, to be in any arbitrary combination of up and down states. (For simplicity and definiteness we take them all to be in their up states.). The replicate spin is in a mixed state of up and down states, at equal shares. Thus, the whole system is in a mixed state, with a density matrix having $1 / 2$ on the diagonal and 0 on the off-diagonals. The entropy of the whole system excluding the bath is at this stage

$$
S_{\text {presR }}=\log 2
$$

\subsection{Post-SR}

Having turned on the control to its value of $W$, which in many cases of reality is presumed to be the effect of enzyme activities, the eigenvalues and eigenstates of the whole system are those given by the Schrödinger equation with the Hamiltonian $H$ in section 4. Postulating a thermal bath temperature much lower than the Zeeman energy of the template, when the thermal interaction with bath is turned on, we shall find the system to be in a state in which the template spin is fully up and, with the control $W$ that favours a T-R alignments switched on, the replicate is also oriented towards the up state. Thus in the low lying, occupied states, the replicate abandons (in a statistical sense) the previous mixed state and resides with a probability close to unity in the up state. A precise measure of the probability is the Reduced Density Matrix (RDM) for the TO sub-system, obtained by tracing over the $N-2$ remaining library states. This yields, as the control $W$ is strengthened, values approaching one and zero, respectively, for the probabilities of the pair T-R being in the (up up) and (up down) states. Since the eigenstates are highly, $2^{N}$-fold entangled, the tracing is not trivial and, in order not to interrupt the description of the results, the derivation of the RDM matrix-elements $\rho_{\text {up up, up up }}(\approx 1), \quad \rho_{\text {up down,up down }}(\approx 0), \quad \rho_{\text {up up, up down }}(=0), \quad \rho_{\text {down up,up down }}(=0)$ is explained in the supplementary information (SI). Its theorems may have wider applications outside the present spin manifold. 
It needs to be stressed that the "alignment" found by the spin-formalism does not proxy for orientational alignment or spatial contiguity, but only a correspondence in the (two-dimensional) Hilbert spaces of the spins. Thus, the two organs represented by $\mathrm{T}$ and $\mathrm{R}$ may be spatially quite detached from each other and be reacting independently for any eventual external stimulations. Such reactions are outside the scope of this paper.

Observationally more accessible than the probabilities is the entropy (here, the post-SR reduced entropy of the TR sub-system and denoted by $S$ ), since the entropy reduction in TO must be compensated over by an increase of entropy in the surrounding, which is manifest by its heat gain. Essentially, this is the basis of the "Theory of Dissipative Structures" propounded by Prigogine [25], elsewhere expressed by the requirement that for the functioning of living systems free energy must be externally supplied to them [19] [26].

In the following plot (Figure 2) we plot the post-SR entropy for the TR sub-system (full lines). To obtain this, we first calculate the reduced occupation probabilities $R P_{i}$ of the four possibilities ( $\left.i=\uparrow \uparrow, \uparrow \downarrow, \downarrow \uparrow, \downarrow \downarrow\right)$ for the TR spins by tracing the computed occupation probabilities over the states of the other spin probabilities and then calculate the entropy as,

$$
S \text {, entropy }=-\sum_{i=1,2,3,4} R P_{i} \log R P_{i}
$$

From the figure it is apparent that as the control $W$ increases the TR entropy decreases, approaching the $S=0$ limit appropriate to a pure state (that of thermodynamically full alignment) for large $W$. Following Nigmatullin and Prokopenko [4] we next compute a quantity, there named "Efficiency", $\eta$ of the SR as the change of entropy relative to the change of control. With our dimensionless parameters this reads as

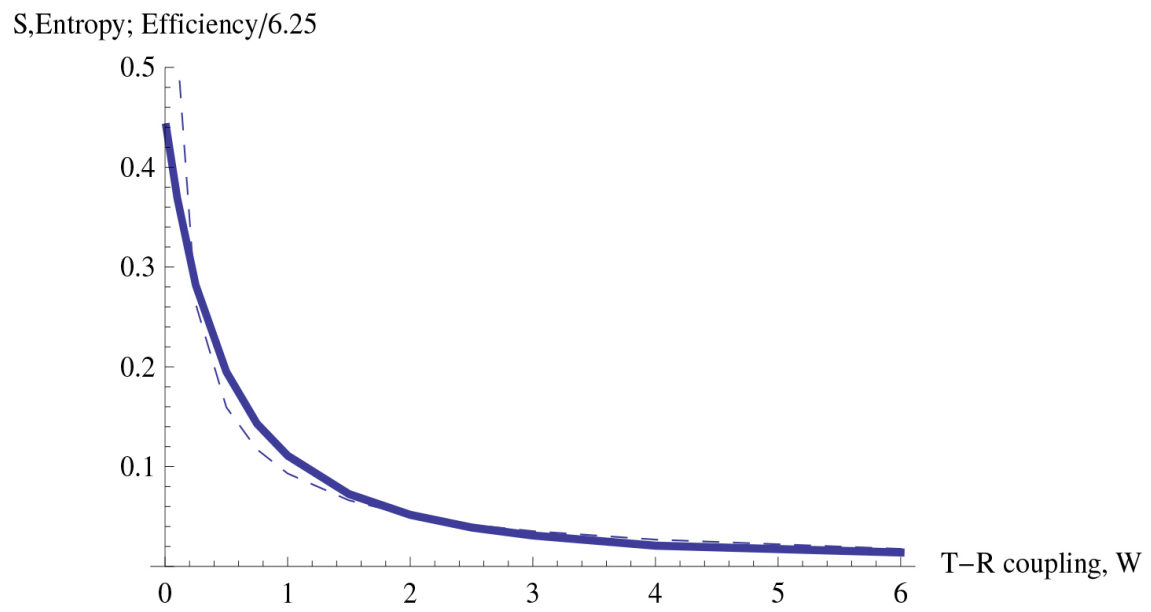

Figure 2. Full line: Post-SR reduced entropy $S$ of the aligned template-replicate spin-pair, as function of the alignment-inducing coupling strength ( $W$, energy units). The asymptotic $S=0$ signifies full alignment (both spins up). Other parameters as in the text; the curve is insensitive to variations in the large value of the template stabilization constant $h_{T}>6$. Broken line: Efficiency (defined in the text Equation (14), downscaled by 6.25). 


$$
\eta=\frac{S_{\text {presR }}-S}{W}
$$

In the figure the efficiency (drawn by broken lines) is plotted; however it is downscaled by a factor of 0.16 , and this so as to bring it into (near) coincidence with the entropy $S$ plotted by full lines. Postulating an exact coincidence for lrge values of $W$ (which are here of interest), one arrives at the following empirical relation for the post-SR entropy:

$$
S=S_{\text {presR }} /(1+6.25 W)
$$

The generality of this relation is not obvious, but it represents a predictive achievement of the theory, to be tested experimentally. The terms "Efficiency" and "work" are used here due to their previous usage in [4], but in the present context they do not have the usual connotations. (Thus, here the "Efficiency" may exceed 1.)

The decrease of T-R entropy, signifying an enhancement of order must be balanced by an increase of entropy exterior to T-R, as required by general thermodynamic principles and ingrained in Prigogine's theory of "dissipative structures". Where does this increase occur? Remarkably, not in the rest of the spin-library, but outside it, in the thermal bath, in line with the general considerations of [34] [35]. This result is shown in the adjacent Figure 3, in which a slow but steady decrease of the combined reduced entropy of the exterior library spins is evident.

\section{Replicativity}

The numerical interplay between the control parameter $W$ of the bilinear T-R term and the "binding energy", linear, Zeeman term strength parameter $h_{R}$ of the replicate is displayed in Figure 4. In this the reduced density of the replicate is contour-plotted with $W$ the abscissa and $h_{R}$ the ordinate. The replicate density

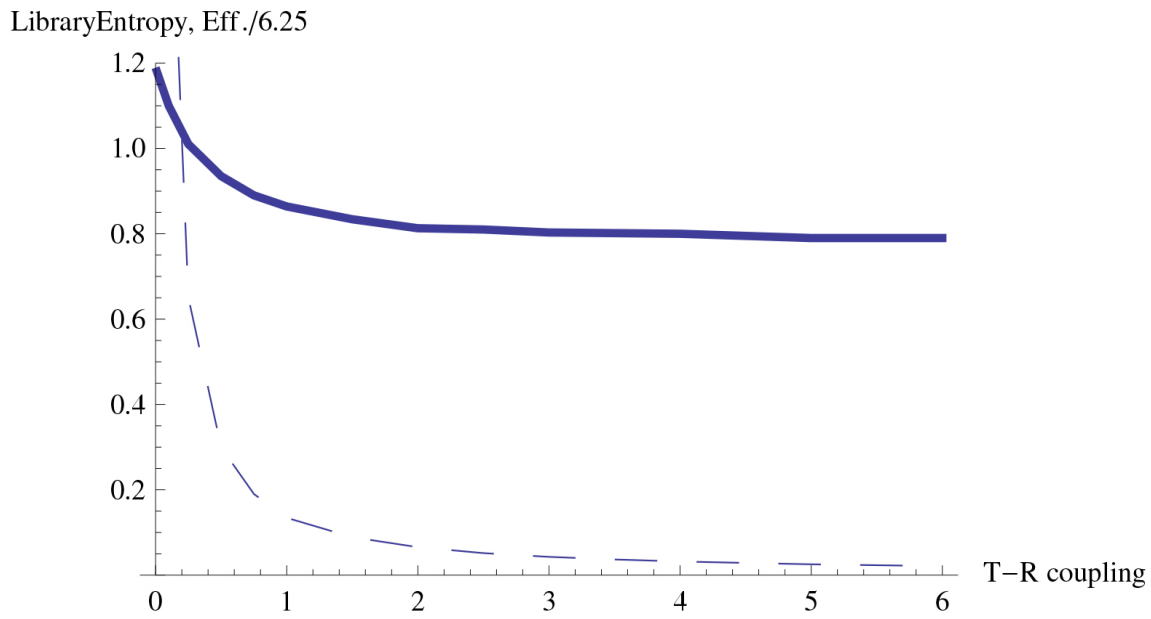

Figure 3. Full line: Combined post-SR reduced entropy of the rest of the spins in the Library, as function of the alignment-inducing coupling strength $W$.). Broken line: Efficiency (defined in the text, Equation (14), downscaled by 6.25). 


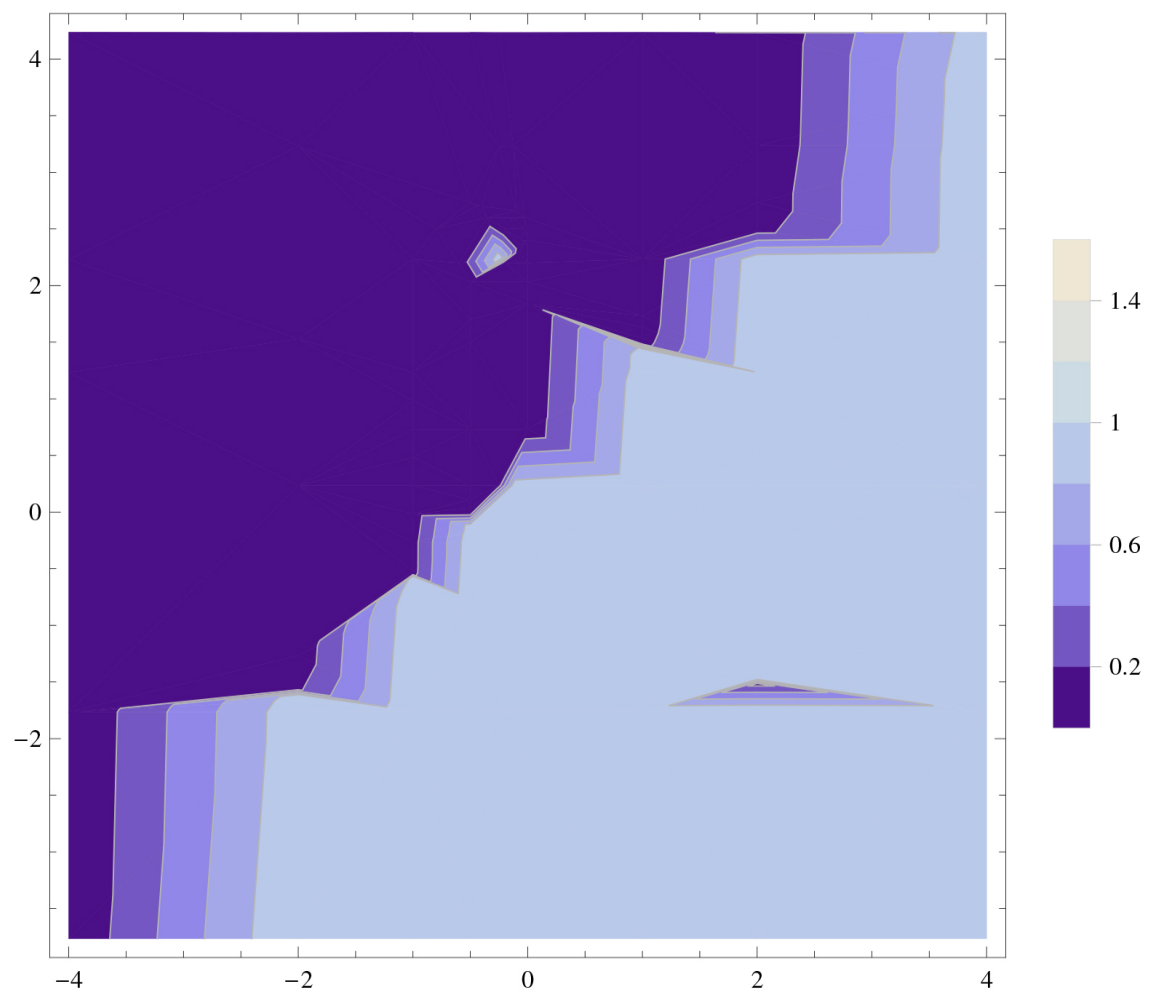

Figure 4. Contour plot depicting the reduced density (occupation probability) for the upper state of the replicate with the control $(W)$ the abscissa and the replicate self-energy $h_{R}$ the ordinate (both in our energy units). Inside the light blue region the upper state occupation probability approaches 1 (alignment with the template); in the dark blue region it tends to zero (anti-alignment). (The colour bar is relevant only between 0 and 1 . The MATHEMATICA program for the figure is available upon request to the author).

is strongly demarcated, with the light blue region (in the right-lower part) representing alignment (replicating capability) between $R$ and $T$ and dark blue region (in the left-upper part) indicating anti-alignment, in the lowest energy state. From the graph, formally for the coefficient $\mathrm{W}$ entering the Hamiltonian as $-W \sigma_{z}^{T} \sigma_{z}^{R}$,

$$
W>h_{R}
$$

is the requirement for alignment, a criterion that is clearly favoring a strongly attractive control. Below this line the upper state density of the replicate tends to be unity alike to the template, while above the line it tends to vanish. The diagonal is a bifurcation line between the aligning and anti-aligning tendency of the ground state and on it the two lowest lying states with opposing alignment tendencies are energetically co-degenerate, implying an entropy increase. (The zig-zag nature of this line is a numerical artefact.) In the immediate vicinity of the line, the reduced replicate density is not yet close to 1 or 0 , this is only the case further inside the respective regions. Speculatively, one may associate the bifurcation line with an inherent instability ("criticality" in Statistical Physics terminology), encountered in some, and perhaps several, biological systems. 
The relatively sharp transition between alignment and anti-alignment is a quantum effect; in a classical description the control term would only gradually affect the statistic distributions of the spin orientation. Whereas the alignment changes dramatically across SR, the weights (RDM values) of the constituents in 2 b' vary only minimally by about 5 percent, a property in line with their presumed enzymatic status.

\subsection{Animate vs. Non-Animate}

Figure 4 suggests, in the context of SR and the limitation of the two spin model, the following criteria as desiderata for the above dichotomy (not excluding further distinctions). The upper right quadrant of the figure is of interest (the rest having been shown only for completeness.) This shows that for SR to occur (i.e., to be below the diagonal line) the control parameter $W$, promoting the alignment of the replicate with the template, must exceed the positive value of the replicate's self energy parameter (which favors non-alignment). In terms of the earlier description of the model in this paper, this requires a strong (bilinear) interaction between $\mathrm{T}-\mathrm{R}$ and the surroundings, as also a weak $\mathrm{R}$ self-energy. The latter may be understood as a relative propensity of the replication candidate for changes (of structure, form, isomerisation, function, etc.), contrasting with non-animate entities that are as a rule fixated relative to their properties. In a terminology of [28] $h_{R}$ is the "durability" of the replicate and, in line with what is found in that paper, its decrease (that is, moving vertically downwards in the figure) favors SR. Changes in the "durability" of the template do not substantially alter the figure. Clearly, not every system satisfying the above criteria will be "biological": in particular, they do not make allowance for the multiply layered sub-conformations [36], belonging to a particular conformation, making the latter a "mixed" rather than a "pure" quantum state (as a single spin state). Yet they provide a direction to which the present SR analysis points.

It may be objected (by regard to the lower half of the figure), that alignment arises also from a negative replica self-energy $\left(h_{R}<0\right)$ irrespective of the control's $W$ strength. However, here the alignment is not due to an SR process, but to the statistical preponderance of the low energy state for the replicate, prior to the SR process.

\subsection{Neglect of Excited States}

At this point it is appropriate to examine the validity of the assumption that the system resides exclusively in its ground state, equivalent to the assumption of the scaled temperature $k T$ low on the relevant energy scale. Our results show that in the parameter regime of strong replicativity, the second lowest level lies at cca. 0.3 in our energy units above the ground level. The stability of the template (meaning that calculationally it stays after the interactions in its low energy state) is due to its large Zeeman term energy-spread $2 h_{T}$, which obtains at the energy value of 10 in our units (though most of the calculations were made with 
a larger energy spread of 48). We may associate the stability with the actual, real life lifetimes of biological entities. These vary over several orders of magnitude; a lifetime at the short end is that of red blood cells $\tau_{R B}$, namely cca. 10 days, or about $10^{7}$ seconds. Assuming a Marcus-type decay or disintegration process with an attempt frequency $v=10^{14} \mathrm{~s}^{-1} \quad$ [19], one may write

$$
\tau_{R B}=v^{-1} \mathrm{e}^{2 h_{T} / k T}
$$

which leads to $k T=\frac{10}{48.3}<0.3$ providing some justification for the neglect of higher lying states.

\section{Conclusions}

The process of living systems with regard to their self-reproductive capacity has here been given a quantum description, differing from (but not replacing) those current descriptions which present them as processes in a thermodynamically non-equilibrium setting. Admittedly, the actual complexity of biological reality has here been drastically reduced by representation of the manifold components through a single spin or qubit and the multiple properties by a single spin direction. It behoves us (and perhaps others) to extend the treatment to models comprising more entities and a variety of properties. A candidate for future treatment might be the self-assembly of a triatomic molecule, acting in some configuration as a template and inducing the assembly of a chosen trio of atoms selected from a large reservoir of atoms.

It may be objected, though, that by identifying the post-SR state with the ground state due to equilibration, thermalization creeps in, somehow which is external to the strictly Hamiltonian formalism of this work. However, according to the Eigenstate Thermalization Hypothesis in some of its formulations [22] [23], the expectation values of the spin alignment (a local observable) will occur autonomously, without thermal contact with an external heat-bath.

\section{Acknowledgements}

I thank helpful suggestions by Professors I. Bersuker, E. Eisenberg, J. P. Klinman and V. Polinger. The help of Prof. A. Yahalom in writing for our joint paper [33] a Mathematica program of the spin-spin Hamiltonian, and here used, and the technical help of N. and T. Kayser are acknowledged.

\section{Data Availability}

The data that support the findings of this study are available from the corresponding author upon reasonable request.

\section{Conflicts of Interest}

The author declares no conflicts of interest regarding the publication of this paper. 


\section{References}

[1] Haken, H. (1983) Synergetics. An Introduction: Nonequilibrium Phase Transitions and Self-Organization in Physics, Chemistry and Biology. 3rd Edition, Springer Verlag, Berlin.

[2] Von Neumann, J. (1966) The Theory of Self-Reproducing Automata. University of Illinois Press, Urbana.

[3] Freitas, M. and Merkle, R. (2004) Kinematic Self-Replicating Machines. Landes Bioscience, Austin.

[4] Nigmatullin, R. and Prokopenko, M. (2020) Thermodynamic Efficiency of Interactions in Self-Organizing Systems.

[5] Cougnon, F.B.L. and Sanders, J.K. (2012) Evolution of Dynamic Combinatorial Chemistry. Accounts of Chemical Research, 45, 2211-2221.

https://doi.org/10.1021/ar200240m

[6] Moulin, E. and Giuseponne, N. (2011) Dynamic Combinatorial Self Replicating Systems. Topics in Current Chemistry, 322, 87-105.

https://doi.org/10.1007/128 2011 198

[7] Alberts, B., Johnson, A., Lewis, J., et al. (2002) Molecular Biology of the Cell. Garland Science, New York.

[8] Carnall, J.M.A., Waudby, C.A., Belenguer, A.M., Stuart, M.C.A. and Peyaralus, J.J.-P. (2010) Mechanosensitive Self-Replication Driven by Self-Organization. Science, 327, 1502-1506. https://doi.org/10.1126/science.1182767

[9] Camazin, S., Deneubourg, J.-L., Franks, N.R., et al. (2001) Self-Organization in Biological Systems. Princeton University Press, Princeton.

https://doi.org/10.1515/9780691212920

[10] Karsenti, E. (2008) Self-Organization in Cell Biology: A Brief History. Nature Reviews Molecular Cell Biology, 9, 255-262. https://doi.org/10.1038/nrm2357

[11] Clixby, G. and Twyman, L. (2016) Self-Replicating Systems. Organic \& Biomolecular Chemistry, 14, 4170-4184. https://doi.org/10.1039/C6OB00280C

[12] Le Vay, K., Weise, L.I., Libicher, K., Mascarenhas, J. and Mutschler, H. (2019) Templated Self-Replication in Biomimetic Systems. Advanced Biosystems, 3, Article ID: 1800313. https://doi.org/10.1002/adbi.201800313

[13] Zelinski, W.S. and Orgel, L.E. (1987) Autocatalytic Synthesis of a Tetranucleotide Analogue. Nature, 327, 346-347. https://doi.org/10.1038/327346a0

[14] Feng, Q., Park, T.K. and Rebek, J. (1992) Cross-Over Reaction between Synthetic Replication Yield Active and Reactive Recombinants. Science, 256, 1179-1180. https://doi.org/10.1126/science.256.5060.1179

[15] Luther, A., Brandsch, R. and von Kiederowski, G. (1998) Surface Promoted Replication and Exponential Amplification on DNA Analogues. Nature, 396, 245-248. https://doi.org/10.1038/24343

[16] Szosztak, J.W. (2012) The Eightfold Path to Non-Enzymatic RNA Replication. Journal of Systems Chemistry, 3, Article No. 2. https://doi.org/10.1186/1759-2208-3-2

[17] Liu, Y. and Sumpter, D.J.T. (2018) Mathematical Modeling Reveals Spontaneous Emergence of Self-Replication in Chemical Reaction Systems. Journal of Biological Chemistry, 293, 18854-18863.

[18] Hordijk, W., Steel, M. and Kauffman, S. (2012) The Structure of Autocatalytic Sets: Evolvability, Enablement, and Emergence. Acta Biotheoretica, 60, 379-392. 
https://doi.org/10.1007/s10441-012-9165-1

[19] Schrödinger, E. (1944) What Is Life? The Physical Aspect of Living Cell. University Press, Cambridge.

[20] Kramers, H.A. (1934) L'interaction entre les atomes magnétogénes dans un cristal paramagnétique. Physica, 1, 182-192. https://doi.org/10.1016/S0031-8914(34)90023-9

[21] Anderson, P.W. (1950) Antiferromagnetism. Theory of Superexchange Interaction. Physical Review, 79, 350-358. https://doi.org/10.1103/PhysRev.79.350

[22] Srednicki, M. (1994) Chaos and Quantum Thermalization. Physical Review E, 50, 888-892. https://doi.org/10.1103/PhysRevE.50.888

[23] Deutsch, J.M. (1991) Eigenstate Thermalisation Hypothesis. Physical Review A, 43, 2046-2049.

[24] Bernal, J.D. (1951) The Physical Base of Life. Routledge and Kegan Paul, London.

[25] Prigogine, I. (1975) Dissipative Structures, Dynamics and Entropy. International Journal of Quantum Chemistry, 9, 443-456. https://doi.org/10.1002/qua.560090854

[26] Dyson, F. (1980) Origins of Life. Cambridge University Press, Cambridge.

[27] Ruelle, D. (2017) The Origin of Life Seen from the Point of View of Non-Equilibrium Statistical Mechanics.

[28] England, J.L. (2015) Dissipative Adaptation in Driven Self-Assembly. Nature Nanotechnology, 10, 919-923. https://doi.org/10.1038/nnano.2015.250

[29] England, J.L. (2013) Statistical Physics of Self-Replication. The Journal of Chemical Physics, 139, 1219-1223. https://doi.org/10.1063/1.4818538

[30] Ruelle, D. (2015) Biology and Nonequilibrium: Remarks on a Paper by J. England, with a Remark by P. Gaspard. European Physics Journal Special Topics, 224, 935-945. https://doi.org/10.1140/epjst/e2015-02437-4

[31] Duboniou, D., Caratzoulas, S., Kalyanaraman, C., Mincer, J.S. and Schwartz, S.D. (2009) Barrier Passage and Protein Dynamics in Enzymatically Catalyzed Reactions. European Journal of Biochemistry, 269, 3103-3112. https://doi.org/10.1046/j.1432-1033.2002.03021.x

[32] Englman, R. and Jortner, J. (1970) The Energy Gap Law for Radiationless Transitions in Large Molecules. Molecular Physics, 18, 145-164. https://doi.org/10.1080/00268977000100171

[33] Englman, R. and Yahalom, A. (2015) Open Systems' Density Matrix Properties in a Time Coarsened Formalism. Foundations of Physics, 45, 673-690. https://doi.org/10.1007/s10701-015-9894-5

[34] Ptaszyncki, K. and Esposito, M. (2019) Entropy Production in Open Systems: The Predominant Role of Intra-Environmental Correlations. Physical Review Letters, 123, Article ID: 200603. https://doi.org/10.1103/PhysRevLett.123.200603

[35] Ptaszyncki, K. and Esposito, M. (2020) Post-Thermalization via Information Stretching in Open Systems.

[36] Ansari, A., Berendsen, J., Browne, S.F., Frauenfelder, H., et al. (1985) Protein States and Protein Quakes. Proceedings of the National Academy of Sciences, 82, 5000-5004. 


\section{Supplementary Information}

\section{Tracing in Binary and "Y-Nary" Digital Representations}

In a previous publication [33] a convenient labelling of the multiple 1/2-spin states was achieved by replacing the ordinary, decimal numbering (in which, e.g., $2^{5}$ is written as 32) with a binary representation, in which the same number is written as [10000] or possibly [000010000], etc. In this representation, the state of the $r$-spin is conveniently registered by having 0 or 1 at the $r$-th position from the right for, respectively the down- or up-state of the state. (While not relevant to the present work we, remark, that for entities or degrees of motion, that have $Y$ rather than two alternative states, with $Y$ being any arbitrary and finite integer, the same idea applies and the combined state would conveniently be represented by an $Y$-nary representation. Thus, for the not uncommon duodecimal representation, each degree of freedom would have entries at each position any one of the figures $1,2, \cdots, 10, X, Y$. That this is not the end of the story, arises from the fact that in most (if not all) programming languages a summation or repeated operation command refers to the decimal representation and this necessitates a shunting between the representations.)

Here we recall that "tracing" is used in the statistical interpretation of quantum mechanics to obtain expectation values for a local operator (meaning one that operates on only one or a few degrees of motion), while summing over all states in the other degrees of motion. Insomuch as tracing also involves a summation [command], it is of interest to have at one's disposal a formula that carries out the tracing for states written in the binary (or Y-nary) representations. The following rule is given for the tracing over a single degree of motion's (namely, the $r^{2}$ th spin's) density of state (also known as the reduced density of state), but other traces follow from this. Astonishingly, the rule is very simple!

Eigen-solutions of the many-spin Hamiltonian matrix in Equation (2) of the text are of the form

$$
|J\rangle=\sum_{\mathbf{n}} C_{\mathbf{n}}^{J}|\mathbf{n}\rangle, J=0, \cdots, 2^{N}-1
$$

where $\mathbf{n}$ runs over the $2^{N}$ combinations of $(0,1)$ (in that order).

\section{Theorem 1}

$$
\begin{aligned}
& \operatorname{Tr}^{r}|0\rangle\left\langle\left. 0\right|^{r}=\sum_{J^{\prime}}\left|C_{\mathbf{n}}^{J^{\prime}}\right|^{2}, J^{\prime}=J \text { if } J \bmod 2^{r}=\left(0, \cdots, 2^{r-1}-1\right)\right. \\
& \operatorname{Tr}^{r}|1\rangle\left\langle\left. 1\right|^{r}=\sum_{J^{\prime \prime}}\left|C_{\mathbf{n}}^{J^{\prime \prime}}\right|^{2}, J^{\prime \prime}=J \text { if } J \bmod 2^{r}=\left(2^{r-1}, \cdots, 2^{r}-1\right)\right.
\end{aligned}
$$

Traces for other local operators than the density operator are treated analogously, with local expectation values inserted in the sums of Equation (19).

We next formulate the problem in more general terms, as an algorithmic exercise, and give a solution in Theorem 2.

$N$ and $Y$ are finite integers. Expressions for the numbers $J=0, \cdots, Y^{N}-1$ in a $Y$-nary system of digits, in a correspondence with a binary or decimal system, can be written as 


$$
J=\left\{I_{N} I_{N-1} \cdots I_{r} \cdots I_{1}\right\}, I_{r}=0, \cdots,(Y-1), \text { for all } r=1, \cdots, N
$$

(please note that $r=1$ is rightmost and $r=N$ is leftmost). (Example with $N$ $=4, Y=3: 11=\{0102\}$.) The problem is to seek a rule as to which $\rho_{s}$ to include in the trace-sum for a given $I_{r}=X$, i.e. which $\rho$ s contain $X$ at $r$ position, with whatever entries at other positions.

\section{Theorem 2}

(Solution of the problem, expressed as a constructive procedure.)

For position $r(=1, \cdots, N)$ arrange all $J\left(0, \cdots, Y^{N}-1\right)$ 's in bunches of size $Y^{r-1}$ numbered $1, \cdots, Y^{N-r+1}$ The trace for any $I_{r}=(0, \cdots, Y-1)$ is then over all $J$ values, starting at the $I_{r}+1$ 'th bunch and continuing to other bunches skipping over them $Y-1$-times. (E.g., for $Y=3, I_{r}=4$ include all $\rho_{s}$ in the fifth, eights, etc. bunch)

\section{Proof}

When $I_{r}$ first appears $J=I_{r} * Y^{r-1}$ (thus in the binary system $\{01000\}=8=1 * 2^{4-1}$ ) and likewise, when $I_{r}+1$ first replaces $I_{r}$, $J=\left(I_{r}+1\right) * Y^{r-1}$, the difference is $Y^{r-1}$ and this is the length of the bunch between them, such that $I_{r}$ is at position $r$. This is also the bunch length, when there are non-zero digits to the left of $r$ and then one meets again $I_{r}$ after $Y-1$ bunches, those in which the digit at position $r$ differs from $I_{r}$.

Illustration: In the binary system at position $r=1$ even and odd digits alternate, while at the last position $r=N$ the first half of $2^{N} J$ 's have 0 's and the second half has 1's.

\section{Corollary}

In many appplications, the number of states differ for different degrees of motion, e.g., in a discretization of radial and angular degrees of motion. Then the bunch length at the $r^{2}$ th position is $Y_{r-1} Y_{r-2} \cdots Y_{1}$, with skipping $\rho_{s} Y_{r}-1$ times over. 